\title{
BMJ Open Evidence characterising skills, competencies and policies in advanced practice critical care nursing in Europe: a scoping review protocol
}

\author{
Gudrun Kaldan, ${ }^{\oplus 1}$ Sara Nordentoft, ${ }^{\oplus 2}$ Suzanne Forsyth Herling, ${ }^{\oplus 3}$ \\ Anders Larsen, ${ }^{\circ}$ Thordis Thomsen, ${ }^{\oplus 5}$ Ingrid Egerod ${ }^{\oplus 6}$
}

To cite: Kaldan G, Nordentoft S, Herling SF, et al. Evidence characterising skills, competencies and policies in advanced practice critical care nursing in Europe: a scoping review protocol. BMJ Open 2019;9:e031504. doi:10.1136/ bmjopen-2019-031504

- Prepublication history and additional material for this paper are available online. To view these files, please visit the journal online (http://dx.doi. org/10.1136/bmjopen-2019031504).

GK and SN contributed equally.

GK and SN are joint first authors.

Received 07 May 2019

Revised 02 August 2019

Accepted 20 August 2019

Check for updates

(C) Author(s) (or their employer(s)) 2019. Re-use permitted under CC BY-NC. No commercial re-use. See rights and permissions. Published by BMJ.

For numbered affiliations see end of article.

Correspondence to

Gudrun Kaldan;

gudrun.kaldan.01@regionh.dk

\section{ABSTRACT}

Introduction The management of critically ill patients is challenged by increasing population age and prevalence of comorbid diseases. High-quality intensive care nursing practice is imperative to accommodate these issues. The roles of the nurse practitioner (NP) and the acute care NP have existed for decades in the USA, Canada and Australia but are still evolving in Europe. Some European countries have introduced the advanced practice nurse (APN), but the current standard of the advanced level of nursing is variable and consensus regarding the framework, role and definition is lacking. Literature and evidence are sparse as well. Identification of skills and competencies required for the APN is warranted. Mapping skills and competencies will enable future educational harmonisation and facilitate mobility of the advanced-level intensive care nursing workforce across Europe. The aim of our scoping review is to identify literature describing skills, competencies and policies characterising advanced nursing practice in intensive care across Europe.

Methods and analysis We will apply a five-stage scoping review methodology with a comprehensive systematic literature search as outlined by Arksey and O'Malley. In collaboration with a research librarian, we will search nine interdisciplinary databases and grey literature for publications originating in European countries in 1992-2018. Using a two-stage screening process with Covidence to remove duplicates, we will first scan the title and abstract and then perform full-text review to determine the eligibility of the papers. Qualitative content analysis will be used to chart the data.

Ethics and dissemination Our study is a part of the European Union-funded INACTIC project (International Nursing Advanced Competency-based Training for Intensive Care) with the overall aim of developing a common European curriculum for advanced practice critical care nursing. Results from this scoping review mapping the evidence of APNs in Europe will be presented at national and international conferences and published in a peer-reviewed journal.

\section{INTRODUCTION}

The management of critically ill patients is challenged by increasing population age, greater prevalence of comorbid diseases
Strengths and limitations of this study

- The scoping review will identify relevant literature answering a comprehensive research question and delivering important educational framework information for curriculum development in advanced-level critical care nursing.

- The scoping review will identify gaps in existing evidence and provide information regarding domains with insufficient knowledge before outlining a new curriculum.

- The scoping review is challenged by variable roles and definitions of advanced practice nurse across Europe.

- The scoping review is limited by the inclusion of publications in English, Danish, Swedish and Norwegian to the exclusion of other European languages.

and significant advances (and costs) associated with medical science. ${ }^{1}$ A more advanced level of nursing practice is needed to accommodate future changes and to optimise management of critically ill patients. Advanced-level nursing has existed in other areas of nursing and other parts of the world, but only a few European countries have developed and implemented a curriculum for advanced practice in critical care nursing and the existing programmes lack consistency. ${ }^{23}$

In the 1960 s, the shortage of primary care physicians in the USA resulted in the establishment of an expanded role in clinical nursing. ${ }^{2}{ }^{4}$ After the role was established and acknowledged, it was applied in more settings and countries. ${ }^{5}$ Due to variation of terms, roles and definitions the advanced practice nurse (APN) was adapted as an umbrella term': 'It should be noted that the term advanced practice nurse is an umbrella term for expanded practice and encompasses a variety of roles, but advanced practice nurse is also used as the actual 
title for expanded nursing roles in some countries'. We will use this term.

In 1999, the Bologna Process (or Copenhagen processes) upgraded basic nursing education in Europe from a hospital diploma to a bachelor's degree and introduced postgraduate degrees in nursing. ${ }^{6-8}$ There is still a need, however, for standardisation of the educational level of specialised nurses. ${ }^{29}$ Standardised advanced practice positively impacts patient safety, ${ }^{10}$ decreases adverse events, ${ }^{1-13}$ prevents severe burnout syndrome, ${ }^{14-17}$ reduces attrition rates $^{18}$ and minimises deskilling of nurses that meet barriers to performing at their level of qualification. ${ }^{19}$ Presence of APNs in emergency and intensive care units has a positive impact on patient safety, ${ }^{20}$ patient experience and quality of care, ${ }^{21}$ length of stay, time to consultation/treatment, mortality, patient satisfaction and cost. ${ }^{1}$ APNs have been shown to promote staff knowledge, skills and competencies, quality of work life, distribution of workload and teamwork, while also contributing to the achievement of organisational priorities and targets and development of policy. ${ }^{18}$

The main challenge to mapping advanced practice nursing is international inconsistency in terms, competencies, and education and curricula. The most common terms are: nurse practitioner (NP) used in Australia, Belgium, Canada, the Netherlands, New Zealand, Sweden, the UK and the USA; $A P N$ used in Ireland, Switzerland, Singapore and South Korea; and finally, clinical nurse specialist (CNS) and nurse specialist (NS) used in Austria, France, Greece, Italy and Spain (NS, only in the following specialities: midwifery, mental health, occupational health, paediatrics, geriatrics, family and community nursing, and medical/surgical nursing). ${ }^{22} 23$ Due to the many roles and titles used, the definitions also vary. ${ }^{23}$ The International Council of Nurses (ICN) defines APN as follows: 'A Nurse Practitioner/Advanced Practice Nurse is a registered nurse who has acquired the expert knowledge base, complex decision-making skills and clinical competencies for expanded practice, the characteristics of which are shaped by the context and/or country in which s/he is credentialed to practice. A master's level degree is recommended for entry level' ${ }^{24}$

A survey of critical care education programmes conducted in intensive care units (ICU) in 24 countries across Europe identified considerable variation in eligibility requirements, modes of assessment and examination, and duration of the programmes (ranging from 30 days to 24 months) ${ }^{25}$ Furthermore, the survey presented 22 countries with challenges in providing continuous education and six countries lacking access to educational resources. Physicians (intensivists) are similarly challenged by variation in the level of education in intensive care medicine across Europe, leading to the development of the Competency-Based Training for Intensive Care across Europe (CoBaTrICE) programme. ${ }^{26}$ The European Union (EU) Policy for Education and Training emphasises the necessity of a comprehensive lifelong learning strategy with transferable and relevant learning outcomes. ${ }^{27}$
With expanding APN standards of practice worldwide, an agreement regarding the specific competency requirements is necessary. ${ }^{28}$ Skills and competencies have been mapped in different ways and it is important that the results are applied to educational programmes. Moreover, there is a need for a joint European curriculum. Mapping skills and competencies pave the way for harmonisation of APN education. The aim of our scoping review is to identify literature describing skills, competencies and policies characterising advanced nursing practice in intensive care across Europe.

\section{METHODS AND ANALYSIS}

The scope of our survey is broad, including skills, competencies and policy. A scoping review is relevant to summarise the breadth of evidence as it 'addresses an exploratory research question aimed at mapping key concepts, types of evidence, and gaps in research related to a defined area or field by systematically searching, selecting, and synthesizing existing knowledge ${ }^{29}$ A scoping review has the potential to clarify elements of our multifaceted topic of advanced nursing practice by integrating a range of study designs and types of literature, which would not be feasible in systematic reviews. Other advantages are: 'Scoping studies are concerned with contextualizing knowledge in terms of identifying the current state of understanding; identifying the sorts of things we know and do not know; and then setting this within policy and practice contexts' ${ }^{30}$ We will follow the five methodological stages outlined by Arksey and O'Malley, and further developed by Levac et al. and Peters et al. ${ }^{30-32}$ The framework includes the following stages: (1) identifying the research question, (2) identifying relevant studies, (3) study selection, (4) charting the data and (5) collating, summarising and reporting the results.

The scoping review is a part of the EU-funded INACTIC study (International Nursing Advanced Competency-based Training for Intensive Care) with the overall aim of developing an international set of competencies, with underpinning evidence base, for advanced-level intensive care nurses.

\section{Stage 1: identifying the research question}

The scoping review will use a broad research question: What characterises skills, competencies and policy in advanced nursing practice in intensive care in Europe? We will categorise our findings in relevant domains used in existing APN curricula and the CoBaTrICE competencies. ${ }^{26}$ The CoBaTrICE domains include: for example, diagnosis, disease management, comfort and recovery, professionalism, practical procedures, and so on. We will define skills and competencies as they are defined by $\mathrm{ICN}^{33}$ : "Competencies refer to the effective application of a combination of knowledge, skill and judgement demonstrated by an individual in daily practice or job performance. In nursing definitions, there is a wide-ranging agreement that, in the performance of nursing roles to the standards required in employment, competence 
reflects the following: (1) knowledge, understanding and judgement; (2) a range of skills cognitive, technical or psychomotor and interpersonal and (3) a range of personal attributes and attitudes".

\section{Stage 2: identifying relevant studies}

The search strategy will be comprehensive. We will identify relevant studies by conducting a literature search in collaboration with a research librarian at the Copenhagen University Hospital Rigshospitalet. We will search electronic databases and reference lists, hand-search and include relevant organisations. The study group will conduct preliminary searches to identify keywords and terms for APNs in Europe. The search terms will be guided by our research question. Our search strategies are guided by the PICO framework (Population, Intervention, Comparison, Outcome) using, in this case, the mnemonics P: population, C: concept and C: context. ${ }^{34}$

\section{Population}

Studies referring to APN or equivalent/corresponding to the individual country of reference, and their highest ICU nurse/critical care nurse education. Titles for expanded nursing roles in ICU are different across Europe; therefore, we will include studies describing advanced critical care practitioner, advanced nurse practice, APN, NS, NP and nurses specialised or expert in critical or intensive care.

\section{Concept}

The focus will be on studies describing education, qualifications, knowledge, curriculum, competencies and skills required for nurses working in advanced critical care. We will also include texts describing national and international APN framework policy.

\section{Context}

The context involves any details regarding the specific setting in level I, II and III intensive or critical care units using APNs.

\section{Types of studies and databases}

We will include all types of study designs. We will conduct a systematic literature search of peer-reviewed articles in the following databases: PubMed, OVID Embase, OVID PsycINFO, EBSCO CINAHL, Cochrane, SweMed, Scopus, ERIC and Social Sciences Citation Index. (Search strategy is attached as online supplementary file.). Concerning the grey literature, we will search OpenGrey, MedNar and ProQuest Dissertations \& Theses Global. Since the first group of NPs from the UK completed their educational programme in 1991, we will limit the literature search to 1992-2018. ${ }^{24}$

\section{Data management}

The computer software programme Covidence will be used for data management, such as identification of duplicates, screening imported studies and maintaining the structure of the process (www.covidence.org). We will use the Preferred Reporting Items for Systematic Reviews and Meta-Analyses flow chart to document our search. ${ }^{35}$

\section{Stage 3: study selection}

Prior to the screening, we will make a random screening test in records retrieved in PubMed, to ensure reliability. We will have to have $>80 \%$ agreement between two reviewers (GK and $\mathrm{SN}$ ). If this goal is not achieved, we will refine and adjust inclusion/exclusion criteria. We will consider the title/abstract screening process to be an iterative process with regular discussions to reach consensus and determine if data are consistent with our research question and objectives. We will schedule meeting with the study group to discuss study selection. Concerning screening of full text articles, two reviewers (GK and SN) will independently review all studies/texts for inclusion. In case of eligibility disagreements, the studies will be re-read and discussed and a third researcher $(\mathrm{SFH})$ will serve as an arbitrator.

\section{Initial inclusion criteria}

- Studies concerning nurses with an APN education equivalent/highest/most or studies concerning ICU/ critical care nurse education corresponding to the individual country of reference in Europe.

- Studies referring to APNs working in an ICU with care of adult patients ( $\geq 18$ years) in Europe.

- Studies describing skills and/or competencies required for an APN in Europe.

- Studies describing policies related to APNs in Europe.

- Studies where the first author is affiliated with a European country.

- Studies related to 50 European countries: 28 EU-member and 22 non-EU-member countries.

\section{Initial exclusion criteria}

- Studies describing a mixed study population of basic nurses, intensive care nurses, APNs, physicians and healthcare professionals making it impossible to discriminate findings specific for APNs.

- Studies describing a setting of paediatric, anaesthesia and recovery ICU.

- Studies or documents not published in English, Danish, Swedish or Norwegian languages.

\section{Stage 4: extraction and charting the data}

We will develop a data extraction tool for reviewing studies and for discussion of variables for extraction. ${ }^{30-32}$ If possible, we will classify findings into two groups: skills/ competencies and policy. Expected extraction fields:

- Author(s).

- Year of publication.

- Source origin/country of origin.

- Aims/purpose/domains.

- Study population and sample size (if applicable).

- Research methodology.

- Intervention type and comparator (if applicable).

- Skills/competencies/knowledge/indicators.

- Duration of the intervention (if applicable). 
- How outcomes are measured.

- Key findings that relate to the review question.

This stage will be an iterative process, continually extracting data and updating our data extraction form. GK and SN will independently extract data from the first 10 included studies and discuss the data charting form and whether data extraction is consistent with our objectives. A qualitative content analysis will be considered for synthesising and analysing qualitative findings. ${ }^{36} 37$

\section{Stage 5: collating, summarising and reporting the results}

We will break this stage into three distinct steps. ${ }^{31} 1 a$. An analysis including descriptive numerical summary analysis of skills/competencies and policy regarding APNs will be carried out, using content or qualitative thematic analysis. ${ }^{37} 18$. We will report our results and produce an outcome referring to our research question. Results and key findings will be presented in a descriptive/narrative form with figures and tables. 1c. We will assess the purpose of our findings and discuss these in relation to our objectives. We will assess implications for APN practice and policy in Europe, and how our findings will feed into the next step in the INACTIC study. Lastly, we will assess implications for future research within advanced practice nursing in intensive care.

\section{Patient and public involvement}

No patient was involved in the design and conception of this study.

\section{TIME SCHEDULE}

\section{Time schedule for INACTIC project Work Package 3, October} 2018-December 2019

- October-December 2018: Initial research and protocol writing.

- January-March 2019: Protocol writing, development of search strategy and data search.

- April-September 2019: Data search, screening, and charting and collating data.

- October-November 2019: Analysis and writing scoping review.

- December 2019: Submission of the final scoping review.

\section{ETHICS AND DISSEMINATION}

The review will inform curriculum development for advanced practice critical care nursing in Europe and map skills and competencies. Findings will contribute to the development of a pan-European curriculum for advanced-level critical care nurses. Mapping skills and competencies will allow for harmonisation of APN education and thereby facilitate mobility of the advanced-level intensive care nursing workforce across Europe. The review contains no personal identifiers and does not need further ethical approval according to the Danish Data Protection Agency and the Danish National Committee on Health Research Ethics. This is accepted by the consortium as data will be handled in Denmark. Results from the scoping review will be presented at national and international meetings and congresses (ESCIM) and published in peer-reviewed journals.

\section{Author affiliations}

${ }^{1}$ Research Unit 7831, Centre for Cancer and Organ Diseases, Copenhagen University Hospital Rigshospitalet, Copenhagen, Denmark

${ }^{2}$ Department of Oncology, Centre for Cancer and Organ Diseases, Copenhagen University Hospital Rigshospitalet, Copenhagen, Denmark

${ }^{3}$ Neuro Centre, Copenhagen University Hospital Rigshospitalet, Copenhagen, Denmark

${ }^{4}$ Department UCSF 9701, Copenhagen University Hospital Rigshospitalet, Copenhagen, Denmark

${ }^{5}$ Department of Anaesthesiology, Copenhagen University Hospital Herlev Gentofte, Herlev, Denmark

${ }^{6}$ Intensive Care Unit 4131, Copenhagen University Hospital Rigshospitalet, Copenhagen, Denmark

Contributors All the authors contributed to the study design. GK and SN performed the review and drafted the protocol. IE, TT, AL and SFH assisted in the review and revised the manuscript. All the authors read and approved the final version of the protocol.

Funding This work is supported by the EU, an EU ERASMUS+ project.

Competing interests None declared.

Patient consent for publication Not required.

Provenance and peer review Not commissioned; externally peer reviewed.

Open access This is an open access article distributed in accordance with the Creative Commons Attribution Non Commercial (CC BY-NC 4.0) license, which permits others to distribute, remix, adapt, build upon this work non-commercially, and license their derivative works on different terms, provided the original work is properly cited, appropriate credit is given, any changes made indicated, and the use is non-commercial. See: http://creativecommons.org/licenses/by-nc/4.0/.

\section{REFERENCES}

1. BFY W, JXY L, WWS T. The impact of the advanced practice nursing role on quality of care, clinical outcomes, patient satisfaction, and cost in the emergency and critical care settings: a systematic review. Hum Resour Health 2017:15:63.

2. Dury C, Hall C, Danan J-L, et al. Specialist nurse in Europe: education, regulation and role. Int Nurs Rev 2014:61:454-62.

3. Heale R, Rieck Buckley C. An international perspective of advanced practice nursing regulation. Int Nurs Rev 2015;62:421-9.

4. Furlong $\mathrm{E}$, Smith R. Advanced nursing practice: policy, education and role development. J Clin Nurs 2005;14:1059-66.

5. Sheer B, Wong FKY. The development of advanced nursing practice globally. J Nurs Scholarsh 2008;40:204-11.

6. Aiken LH, Sloane DM, Bruyneel L, et al. Nurse staffing and education and hospital mortality in nine European countries: a retrospective observational study. Lancet 2014;383:1824-30.

7. Lahtinen P, Leino-Kilpi H, Salminen L. Nursing education in the European higher education area - variations in implementation. Nurse Educ Today 2014;34:1040-7.

8. Collins S, Hewer I. The impact of the bologna process on nursing higher education in Europe: a review. Int J Nurs Stud 2014;51:150-6.

9. Ranchal A, Jolley MJ, Keogh J, et al. The challenge of the standardization of nursing specializations in Europe. Int Nurs Rev 2015;62:445-52.

10. Valentin A, Bion J. How safe is my intensive care unit? an overview of error causation and prevention. Curr Opin Crit Care 2007; 13:697-702.

11. Coopersmith CMet al. The impact of bedside behavior on catheter-related bacteremia in the intensive care unit. Arch Surg 2004;139:131-6.

12. Warren DK, Zack JE, Mayfield JL, et al. The effect of an education program on the incidence of central venous catheter-associated bloodstream infection in a medical ICU. Chest 2004;126:1612-8.

13. Soupios MA, Lawry K. Stress on personnel working in a critical care unit. Psychiatr Med 1987;5:187-98. 
14. Sawatzky J-AV. Stress in critical care nurses: actual and perceived. Heart \& Lung 1996;25:409-17.

15. Chen S-M, McMurray A. "Burnout" in Intensive Care Nurses. J Nurs Res 2001;9:152-64.

16. Poncet MC, Toullic $P$, Papazian $L$, et al. Burnout syndrome in critical care nursing staff. Am J Respir Crit Care Med 2007;175:698-704.

17. Coopersmith CM, Rebmann TL, Zack JE, et al. Effect of an education program on decreasing catheter-related bloodstream infections in the surgical intensive care unit. Crit Care Med 2002;30:59-64.

18. Shields MA, Ward M. Improving nurse retention in the National health service in England: the impact of job satisfaction on intentions to quit. J Health Econ 2001;20:677-701.

19. O'Brien T. Overseas nurses in the National health service: a process of deskilling. J Clin Nurs 2007;16:2229-36.

20. Newhouse RP, Stanik-Hutt J, White KM, et al. Advanced practice nurse outcomes 1990-2008: a systematic review. Nurs Econ 2011;29:230-50.

21. McDonnell A, Goodwin E, Kennedy F, et al. An evaluation of the implementation of advanced nurse practitioner (ANP) roles in an acute hospital setting. J Adv Nurs 2015;71:789-99.

22. Pulcini J, Jelic M, Gul R, et al. An international survey on advanced practice nursing education, practice, and regulation. J Nurs Scholarsh 2010;42:31-9.

23. Bryant-Lukosius D, Spichiger E, Martin J, et al. Framework for evaluating the impact of advanced practice nursing roles. J Nurs Scholarsh 2016:48:201-9.

24. ICN Nurse Practioner/Advanced Practice Nursing Network. Frequently asked questions of the ICN international NP/APN network. Available: http://international.aanp.org/Home/FAQ [Accessed 21 Jan 2019].

25. Endacott R, Jones $\mathrm{C}$, Bloomer MJ, et al. The state of critical care nursing education in Europe: an international survey. Intensive Care Med 2015;41:2237-40.

26. The CoBaTrICE Collaboration. The educational environment for training in intensive care medicine: structures, processes, outcomes and challenges in the European region. Intensive Care Med 2009;35:1575-83.

27. European Policy Cooperation. (ET2020 framework) The strategic framework for European cooperation in education and training ("ET 2020") is a forum that allows Member States to cooperate in building best. Available: https://ec.europa.eu/education/policies/europeanpolicy-cooperation/et2020-framework_en [Accessed 21 Jan 2019].

28. Sastre-Fullana P, De Pedro-Gómez JE, Bennasar-Veny M, et al. Competency frameworks for advanced practice nursing: a literature review. Int Nurs Rev 2014;61:534-42.

29. Colquhoun HL, Levac D, O'Brien KK, et al. Scoping reviews: time for clarity in definition, methods, and reporting. J Clin Epidemiol 2014;67:1291-4.

30. Arksey H, O'Malley L. Scoping studies: towards a methodological framework. Int J Soc Res Methodol 2005;8:19-32.

31. Levac D, Colquhoun H, O'Brien KK. Scoping studies: advancing the methodology. Implementation Sci 2010;5.

32. Peters MDJ, Godfrey CM, Khalil H, et al. Guidance for conducting systematic scoping reviews. Int J Evid Based Healthc 2015;13:141-6.

33. ICN Regulation Serie. Icn framework for competencies for the nurse specialist, 2009. Available: https://siga-fsia.ch/files/user_upload/ 08_ICN_Framework_for_the_nurse_specialist.pdf [Accessed 22 Feb 2019].

34. The Joanna Briggs Institute Reviewer's Manual 2015 edition/ supplement. Methodology for JBI scoping reviews, 2015.

35. Moher D, Shamseer L, Clarke M, et al. Preferred reporting items for systematic review and meta-analysis protocols (PRISMA-P) 2015 statement. Syst Rev 2015;4:1.

36. Elo $\mathrm{S}$, Kyngäs $\mathrm{H}$. The qualitative content analysis process. $J \mathrm{AdV}$ Nurs 2008;62:107-15.

37. Graneheim UH, Lundman B. Qualitative content analysis in nursing research: concepts, procedures and measures to achieve trustworthiness. Nurse Educ Today 2004;24:105-12.

38. Braun V, Clarke V. Using thematic analysis in psychology. Qual Res Psychol 2006;3:77-101. 